\title{
Multiple mycoviruses identified in Pestalotiopsis spp. from Chinese bayberry
}

Fangyong Chen ${ }^{*}$, Zhanxu Pu, Haizhi Ni, Yin Wang and Bangguo Yan

\begin{abstract}
Background: Chinese bayberry (Myrica rubra) is a subtropical fruit crop widely grown in southern China. Twig dieback is a disease of Chinese bayberry caused by Pestalotiopsis spp. and results in great economic losses to Chinese bayberry production. A virus survey was conducted in the population of Pestalotiopsis spp. infecting M. rubra in China. We explored the viral diversity in Pestalotiopsis spp., which may provide resources for further development as biocontrol agents of twig dieback.

Methods: Strains of Pestalotiopsis spp. were isolated from diseased twigs of M. rubra, and cultured on potato dextrose agar for RNA extraction. The total RNA of each strain was extracted, mixed, and used for RNA sequencing. The resulting sequences were deduplicated, annotated, and then used for phylogenetic analysis.

Results: Seven novel viruses were characterized from 59 isolates of $M$. rubra collected from 14 localities in China. Based on the phylogenetic analysis, these viruses were classified into five viral families/orders, Botourmiaviridae, Mitoviridae, Partitiviridae, Tymovirales and Bunyavirales, and one virus, Pestalotiopsis negative-stranded RNA virus 1, which likely belongs to a new viral family.

Conclusions: Metatranscriptomics analysis showed the presence of various mycoviruses in Pestalotiopsis spp. isolated from M. rubra in China. The genomes of eight putative viruses were identified, seven of which were nearly fulllength. Some of these viruses of Pestalotiopsis spp. may have the potential for the biological control of twig dieback of M. rubra.
\end{abstract}

Keywords: Pestalotiopsis spp., RNA-seq, Mycovirus

\section{Introduction}

Chinese bayberry (Myrica rubra) is a subtropical fruit crop with great economic value that is widely grown in southern China. The fruits of M. rubra are not only eaten fresh but also can be processed into various products, such as juice, jam, sweets, and wine [1]. Recently, a disease causing twig dieback of $M$. rubra was found in all major Chinese $M$. rubra producing areas [2, 3]. The disease causes brown spots on the leaves and twigs of the diseased plants during the early stages of infection, and the infected trees experience leaf drop and may die within 1-4 years [2]. The disease spreads quickly and

*Correspondence: cfy17266@126.com

Citrus Research Institute of Zhejiang Province, Taizhou 318026, China is becoming a serious threat to the development of $M$. rubra. Many species of Pestalotiopsis, like Pestalotiopsis mangiferae, $P$. vismiae, $P$. microspora, and $P$. versicolor, were determined to be the causal agents of twig dieback of M. rubra in China [2-4]. Besides being a plant pathogen, Pestalotiopsis spp. are also endophytes in many plant species, representing a huge and largely untapped resource of natural products [5].

Mycoviruses or fungal viruses refer to viruses parasitizing or inhabiting fungi and oomycetes. They are widely discovered in all major groups of fungi. The earliest record of mycoviruses was believed to be the discovery of virus-like particles in diseased Agaricus bisporus [6]. Due to the potential of using mycoviruses for the control of fungal diseases of plants [7] and the original author(s) and the source, provide a link to the Creative Commons licence, and indicate if changes were made. The images or other third party material in this article are included in the article's Creative Commons licence, unless indicated otherwise in a credit line to the material. If material is not included in the article's Creative Commons licence and your intended use is not permitted by statutory regulation or exceeds the permitted use, you will need to obtain permission directly from the copyright holder. To view a copy of this licence, visit http://creativecommons.org/licenses/by/4.0/. The Creative Commons Public Domain Dedication waiver (http://creativeco mmons.org/publicdomain/zero/1.0/) applies to the data made available in this article, unless otherwise stated in a credit line to the data. 
rapid development of sequencing technology, the presence of mycoviruses has been investigated in many different fungi, quickly increasing the number of known mycoviruses [8-12]. Mycoviruses are generally classified according to their hosts, genomic structure, and shape of virus particles, and most of them possess RNA genomes of double-stranded (ds) RNA (Chrysoviridae, Totiviridae, and Paritiviridae, among others), positive single-stranded (+ss) RNA (Alphaflexiviridae, Betaflexiviridae, and Narnaviridae, among others), or negative single-stranded (-ss) RNA (Mymonaviridae). Nevertheless, many mycoviruses remain unclassified [13]. Besides RNA viruses, a few ssDNA viruses have been reported, such as Sclerotinia sclerotiorum [14] and Fusarium graminearum [15].

Next-generation sequencing (NGS) technology is a popular way to explore mycoviral diversity, including sequencing the total RNA that is depleted of ribosomal RNA or small RNAs [8-11]. Many novel viruses have been detected using NGS. For instance, 72 partial or complete genome segments, representing 66 previously undescribed mycoviruses, were identified in the metatranscriptomes of five widely distributed plant pathogenic fungi [10]. Similarly, 34 contigs, representing novel viruses, were identified in the virome of an Australian S. sclerotiorum population [12]. However, no viruses have been reported in the population of Pestalotiopsis spp. Therefore, in this study, we aimed to identify the viral population in Pestalotiopsis spp. infecting $M$. rubra in China by analyzing the total RNA with NGS technology.

\section{Materials and methods}

\section{Isolates and growth conditions}

In 2019, strains of Pestalotiopsis spp. were isolated from diseased twigs of M. rubra collected from 14 localities (Additional file 1: Table S1) in China, and then identified according to the method previously described $[2,3]$. The stock cultures were maintained in potato dextrose agar (PDA) plates at $4{ }^{\circ} \mathrm{C}$. Working cultures were established by transferring stock agar with mycelium onto PDA at $20{ }^{\circ} \mathrm{C}$ for 1 week.

\section{Total RNA extraction and purification}

The total RNA of each strain was extracted from $0.1 \mathrm{~g}$ of fungal mycelium by using a Plant RNA Extraction Kit (Omega, America). The total RNA was stored at $-80^{\circ} \mathrm{C}$ until use. Approximately $2 \mu \mathrm{g}$ of RNA were taken from each sample (59 strains) and then mixed. A mixed RNA sample $(\sim 120 \mu \mathrm{g})$ was sent to Novogene (Beijing, China) for RNA sequencing (RNA-seq).

\section{RNA sequencing and sequence analysis}

Ribosomal RNA depletion (Ribo-Zero rRNA Removal Kit, Illumina, Inc.), library preparations $(\sim 1 \mu \mathrm{g}$ RNA sample, TruSeq RNA Sample Preparation Kit, Illumina, Inc.) and Illumina sequencing (Illumina Novaseq 6000) were performed by Novogene (Beijing, China). The filtered data was spliced from scratch using Trinity software (version: 2.3.3), and the resulting sequences were then deduplicated with cd-hit (version: 4.6). Finally, Diamond software (version: 0.8.22) and the non-redundant protein database in the National Center for Biotechnology Information (NCBI) (https://www.ncbi.nlm.nih. gov/) were used for BLASTx annotation, and the viral sequences were selected. Pairwise sequence comparisons were carried out for both botourmiavirus and mitovirus related sequences, using Sequence Demarcation Tool (SDT) v.1.0 with the MUSCLE alignment option [16].

\section{Confirmation of viral-like contigs}

To confirm the presence of viral-like contigs in the tested strains, the presence of each contig was determined by using RT-PCR with its corresponding primer pairs [12]. The mixed RNA sample was used as template for reverse transcription to cDNA using MMLV reverse transcriptase (TOYOBO Co., Ltd., Osaka, Japan). The cDNA strand was then used as template in PCR to amplify a specific DNA band with the corresponding primer pairs listed in Additional file 1: Table S2, respectively.

\section{Phylogenetic analysis}

Open reading frames (ORFs) and conserved domain(s) were determined by using the ORF Finder program and CD-search in NCBI. Then, the selected amino acid (aa) sequences of viral replicases or other conserved regions were used for the construction of phylogenetic trees with the neighbor-joining (NJ) method and tested with a bootstrap of 1000 replicates to ascertain the reliability of a given branch pattern in MEGA X software [17]. Multiple alignments of the sequences were performed using ClustalX.

\section{Results}

Viral sequences in the metatranscriptome of Pestalotiopsis Fifty-nine Pestalotiopsis strains were isolated form the diseased samples collected from 14 locations (Additional file 1: Table S1). All these strains were determined to be Pestalotiopsis spp. based on our methods previously described $[2,3]$. One sequencing library was constructed from the rRNA-depleted total RNAs of the 59 Pestalotiopsis strains. After the removal of all low-quality reads, $9.2 \times 10^{7}$ reads with lengths $>20$ nt (paired-end) were obtained, and a total of 164,628 contigs were assembled 
by using the software Trinity. A BLAST search of nonredundant protein database identified a total of 8 putative viral contigs (Table 1), possibly belonging to viral families Botourmiaviridae, Narnaviridae, Deltaflexiviridae, Partitiviridae, and the order Bunyavirales. The presence of these putative viral contigs in the Pestalotiopsis strains were confirmed by RT-PCR (Additional file 1: Fig. S1). Through conserved motif analysis, all contigs were found to possess a conserved domain of GDD except -ss viruses.

\section{Botourmiavirus-related sequences}

The fragment c49783_g1 was $2438 \mathrm{nt}$ in length, possessing a large ORF encoding a 653 aa peptide showing homology to the RNA dependent RNA polymerase (RdRp) sequence of Colletotrichum gloeosporioides ourmia-like virus 1 (CgOLV1, QDW80875.1) with an aa sequence identity of $96 \%$. Therefore, this fragment may represent a strain of CgOLV1. The fragment c55068_g1 was 2634 nt long, encoding a putative RdRp of 639 aa, which was $61 \%$ identical to the RdRp sequence of Plasmopara viticola associated ourmia-like virus 44 (QGY72574.1), and it was named Pestalotiopsis botourmiavirus 2 (PBV-2). The fragment c59323_g1 was 2460 nt in length encoding a putative RdRp of 636 aa, namely Pestalotiopsis botourmiavirus 3 (PBV-3). The RdRp of PBV-3 was most homologous to the RdRp sequence of Plasmopara viticola associated ourmia-like virus 81 (QGY72611.1) with aa sequence identity of $39 \%$ (Fig. 1a). Pairwise identity comparisons of PBV-2 and PBV-3 sequences to other known botoumiaviruses demonstrated that PBV-2 and PBV-3 shared $<68 \%$ genome identity with other known botoumiaviruses (Additional file 1: Fig. S2a). In addition, the multiple sequence alignments of the RdRps of CgOLV1, PBV-2, and PBV-3 detected eight conserved motifs that were characteristic of the RdRps of + ssRNA mycoviruses [18] (Additional file 1: Fig. S3). To establish the phylogeny of the three botoumiaviruses with other RNA viruses, a phylogenetic tree was constructed by using the NJ method based on the viral RdRp aa sequences. The results showed that PBV-3 was clustered with the botouliviruses. In contrast, PBV-2 was mostly related to Plasmopara viticola associated ourmia-like virus 44 , likely belonging to the genus Scleroulivirus, while CgOLV1/c49783_g1 was mostly related to magouliviruses and might be a new member in this genus (Fig. 2).

\section{Mitovirus-related sequences}

Two fragments, c61286_g1 (2486 nt) and c61286_g1 (2486 nt), were predicted to encode mitoviral RdRps, namely Pestalotiopsis mitovirus 1 (PMV-1) and Pestalotiopsis mitovirus 2 (PMV-2), respectively. The RdRp of PMV-1 was most similar to Sclerotinia homoeocarpa mitovirus (AAO21337.1), with an aa sequence identity of 49\%, while PMV-2 was most similar to Rhizoctonia solani mitovirus 10 (ALD89102.1), with an aa sequence identity of $44 \%$ (Fig. 1a). Pairwise identity comparisons of PMV-1 and PMV-2 sequences to other known mitoviruses demonstrated that PMV-1 and PMV-2 shared $<69 \%$ genome identity with other known mitoviruses (Additional file 1: Fig. S2b). Multiple alignments based on viral RdRps indicated that RdRps of PMV-1 and PMV-2 contained six

Table 1 Assembled sequences with similarity to previously described viruses

\begin{tabular}{|c|c|c|c|c|c|c|}
\hline Contig number & GenBank ID & $\begin{array}{l}\text { cDNA } \\
\text { Contig } \\
\text { length (bp) }\end{array}$ & Name of putative viruses & Best match ${ }^{a}$ & $\begin{array}{l}\text { aa } \\
\text { identity } \\
(\%)\end{array}$ & Family/Genus \\
\hline c61286_g1 & MW017458 & 2486 & Pestalotiopsis mitovirus 1 & $\begin{array}{l}\text { Sclerotinia homoeocarpa mitovirus } \\
\text { (AAO21337.1) }\end{array}$ & 49 & Mitoviridae \\
\hline c61448_g1 & MW017459 & 2303 & Pestalotiopsis mitovirus 2 & $\begin{array}{l}\text { Rhizoctonia solani mitovirus } 10 \\
\text { (ALD89102.1) }\end{array}$ & 44 & Mitoviridae \\
\hline c49783_g1 & MT925636 & 2438 & $\begin{array}{l}\text { Colletotrichum gloeosporioides } \\
\text { ourmia-like virus } 1\end{array}$ & $\begin{array}{l}\text { Colletotrichum gloeosporioides } \\
\text { ourmia-like virus } 1 \text { (QDW80875.1) }\end{array}$ & 96 & Botourmiaviridae \\
\hline c55068_g1 & MW017456 & 2674 & Pestalotiopsis botourmiavirus 2 & $\begin{array}{l}\text { Plasmopara viticola associated } \\
\quad \text { ourmia-like virus } 44 \text { (QGY72574.1) }\end{array}$ & 61 & Botourmiaviridae \\
\hline c59323_g1 & MW017457 & 2460 & Pestalotiopsis botourmiavirus 3 & $\begin{array}{l}\text { Plasmopara viticola associated } \\
\quad \text { ourmia-like virus } 81 \text { (QGY72611.1) }\end{array}$ & 39 & Botourmiaviridae \\
\hline c43244_g1 & MW017460 & 7667 & Pestalotiopsis deltaflexi-like virus 1 & $\begin{array}{l}\text { Rhizoctonia solani flexivirus } 1 \\
\quad\left(Y P \_009268715.1\right)\end{array}$ & 30 & Unclassifified \\
\hline c48689_g1 & MT925635 & 7009 & $\begin{array}{l}\text { Pestalotiopsis negative-stranded } \\
\text { RNA virus } 1\end{array}$ & $\begin{array}{l}\text { Plasmopara associated mycobun- } \\
\text { yavirales-like virus } 2 \text { (QGY72639.1) }\end{array}$ & 34 & Unclassifified \\
\hline c9266_g1 & MT925634 & 1646 & Pestalotiopsis partitivirus 1 & $\begin{array}{l}\text { Diatom colony associated dsRNA } \\
\text { virus } 14 \text { (BAU79511.1) }\end{array}$ & 49 & Partitiviridae \\
\hline
\end{tabular}

\footnotetext{
a The Genbank accession number for each virus is listed in the parentheses
} 


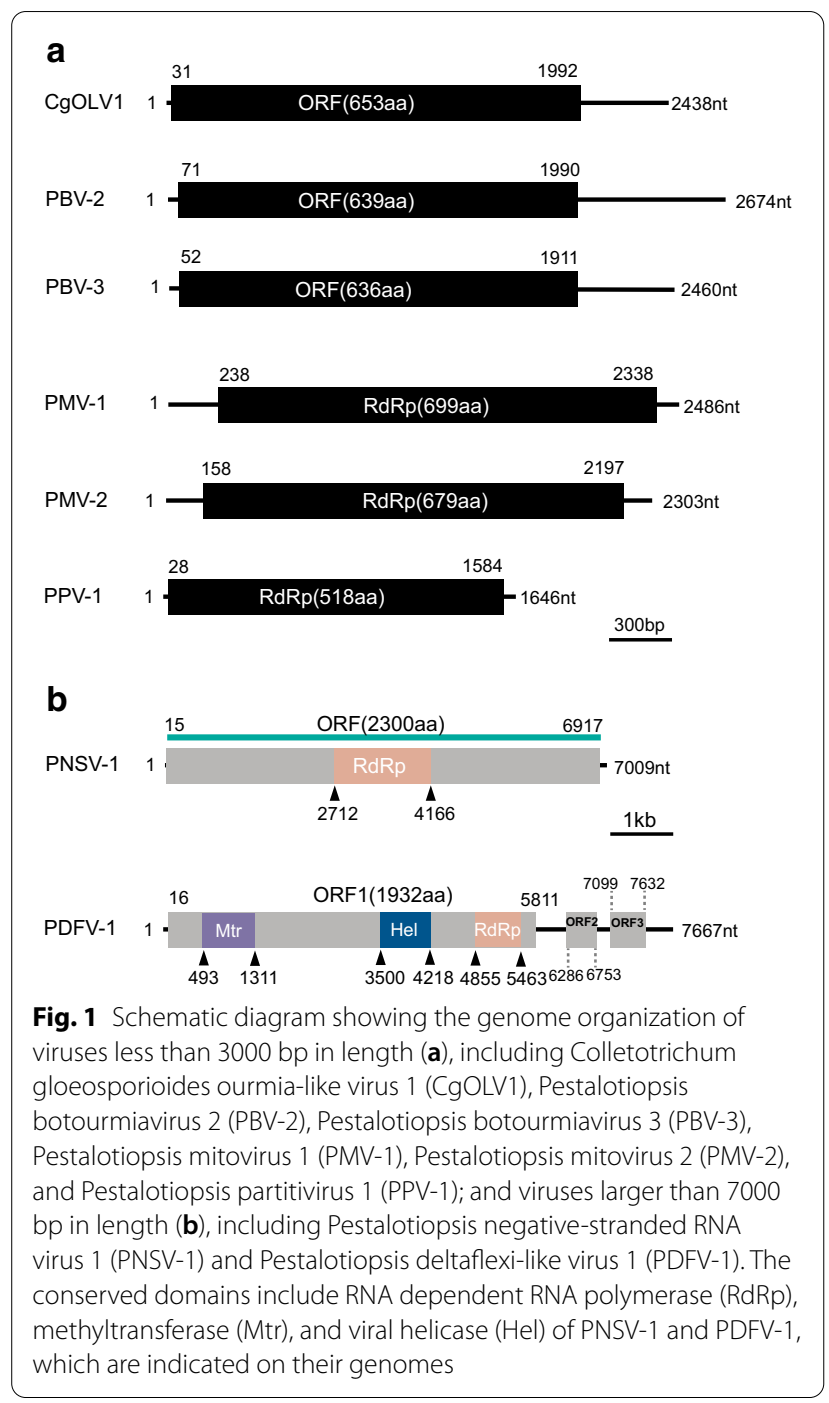

conserved motifs, which is the characteristic of mitoviruses [19] (Additional file 1: Fig. S4). Phylogenetic analysis using the RdRp aa sequences also showed that PMV-1 and PMV-2 were clustered with other mitoviruses based on the NJ method (Fig. 2).

\section{Tymovirales-related sequences}

The fragment c43244_g1 was 7667 nt long and encoded a 1932 aa-long polyprotein, containing a methyltransferase (Mtr) and a viral helicase (Hel) domain (Fig. 1b). BLAST and phylogenetic analysis showed that the closest related virus to this fragment was Rhizoctonia solani flexivirus 1 (YP_009268715.1) with a sequence identity of $30 \%$ at the aa level. Therefore, it was temporarily named Pestalotiopsis deltaflexivirus 1 (PDFV-1). Furthermore, multiple alignments revealed that Mtr domain of PDFV-1 contained six conserved motifs, which were similar to the viral Mtr domains of Sclerotinia sclerotiorum deltaflexivirus 1 (NC_038977), Sclerotinia sclerotiorum deltaflexivirus 2 (NC_040649), and Fusarium graminearum deltaflexivirus 1 (NC_030654). In addition, the Hel domain of PDFV-1 was also similar to viruses in the family Deltaflexiviridae, and the $\mathrm{RdRp}$ domain of PDFV-1 contained six typical viral RdRp motifs similar to other deltaflexiviruses [35, 36] (Additional file 1: Fig. S5). To examine the phylogenetic relationship of PDFV-1 to viruses in Tymovirales, a phylogenetic tree was constructed based on the aa sequence of the polyproteins. The results showed that PDFV-1 was clustered with viruses of Deltaflexiviridae with $100 \%$ bootstrap support (Fig. 3).

\section{Bunyavirales-related sequences}

In addition to + ssRNA viruses, a possible -ssRNA viral segment (c48689_g1) was also identified, namely Pestalotiopsis negative-stranded RNA virus 1 (PNSV-1). The identified PNSV-1 contig was 7009 nt long and putatively encoded a polyprotein of 2300 aa in length, containing an RdRp domain and a Hel domain (Fig. 1b). BLASTp analysis showed that the RdRp was $34 \%$ identical to that of Plasmopara associated mycobunyavirales-like virus 2 (QGY72639.1). The alignment of the RdRps of PfNSV-1, fig mosaic emaravirus, and rose rosette emaravirus indicated that the RdRps possessed five conserved motifs, which were also present in the RdRps of the typical members in Bunyavirales (Additional file 1: Fig. S6). Phylogenetic analysis revealed that PNSV-1 was grouped with Barns Ness serrated wrack bunya/phlebo-like virus 1 with $100 \%$ bootstrap support, which might belong to a novel viral family (Fig. 4).

\section{Partitivirus-related sequences}

According to the BLASTx search, only one fragment (c9266_g1) of 1646 bp in length, encoding a 518 aa long putative RdRp, showing homology to partitiviruses, namely Pestalotiopsis partitivirus 1 (PPV-1) (Fig. 1a). PPV-1 was most closely related to Diatom colony associated dsRNA virus 14 , with the aa sequence identity of $49 \%$. However, no coat protein (CP) sequence of PPV-1 was obtained. Eight conserved motifs were detected in PPV-1, as well as in beet cryptic virus 1, beet cryptic virus 2, beet cryptic virus 3 , and epirus cherry virus, through multiple sequence alignments of the RdRps [20] (Additional file 1: Fig. S7). Phylogenetic analysis showed that PPV-1 was clustered with other deltapartitiviruses, forming an independent branch (Fig. 5).

\section{Discussion}

Twig dieback of M. rubra caused by Pestalotiopsis spp. is a new threat to the production of M. rubra in China [2, 3]. The present study showed that various mycoviruses 


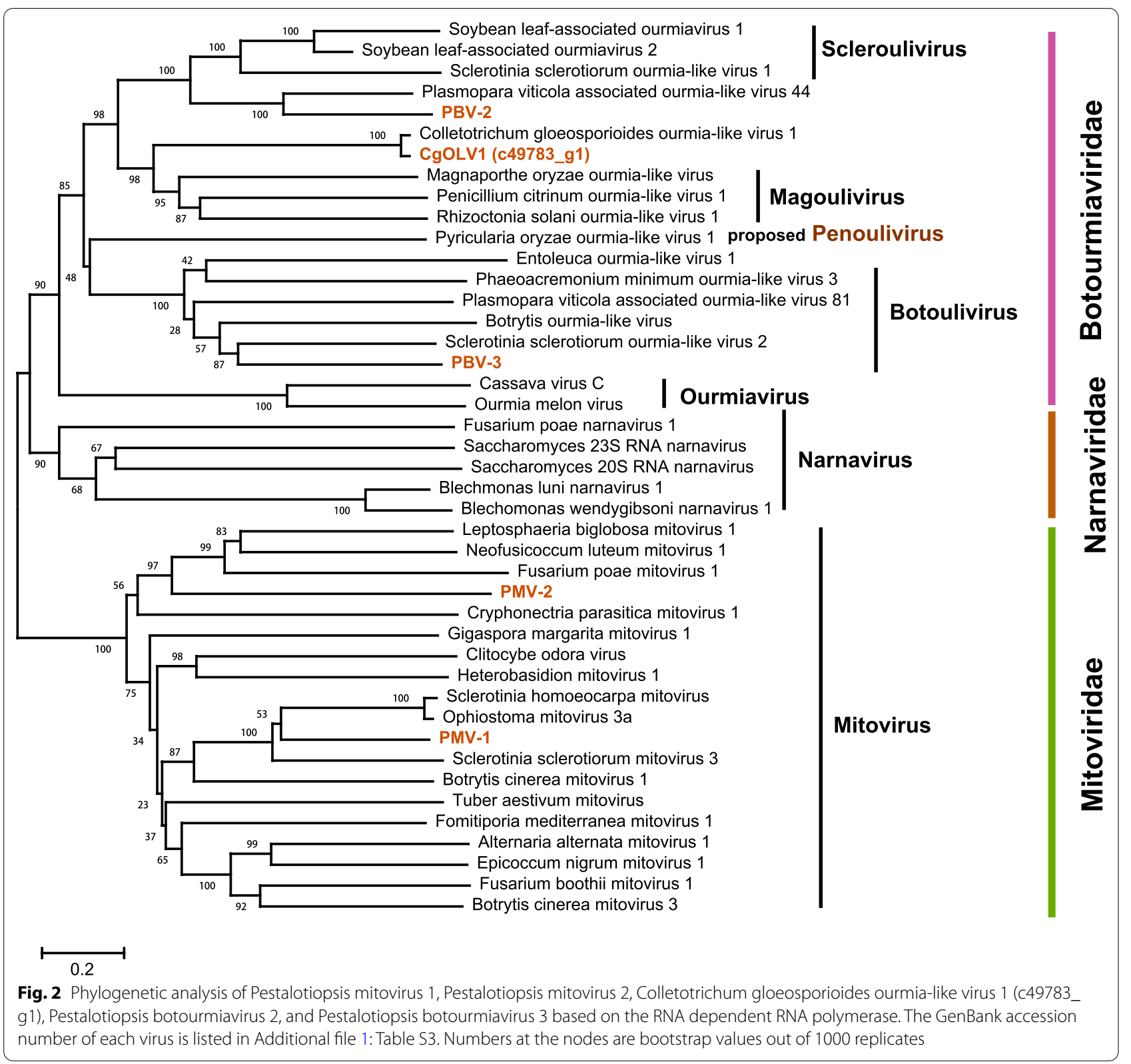

persist in the population of Pestalotiopsis spp. isolated from M. rubra in China. In this study, eight putative viral sequences were identified, many of which were nearly full-length. These viruses could be grouped into five distinct lineages, including Botourmiaviridae, Narnaviridae, Partitiviridae, Tymovirales, and Bunyavirales.

Viruses of the Botourmiaviridae usually possess a large open reading frame, encoding the viral replicase [21, 22], and there were three contigs that might belong to this family. In these viruses, two botourmiaviruses, PBV-2 and PBV-3, were novel and probably belonged to Scleroulivirus and Botoulivirus, respectively. It is of interest that the results showed that CgOLV1 was able to infect two different fungal species. Although the transmission of mycoviruses is generally believed to be restricted by vegetative incompatibility between the donor and receipt strains, viral interspecies transmission has been observed in some cases, such as those from S. sclerotiorum to $S$. minor [23], from Cryphonectria parasitica to C. nitschkei [24], and from Aspergillus niger to A. nidulans [25]. In addition, a few mycoviruses were reported to be present in different fungal hosts. For example, two mitoviruses, OnuMV3a and BcMV1 were detected in different fungal hosts, and BpBV1 may also be widely distributed in populations of B. cinerea, B. squomosa, and S. sclerotiorum $[26,27]$. The -ssRNA virus, $\mathrm{BcMyV} 1$ was detected in 


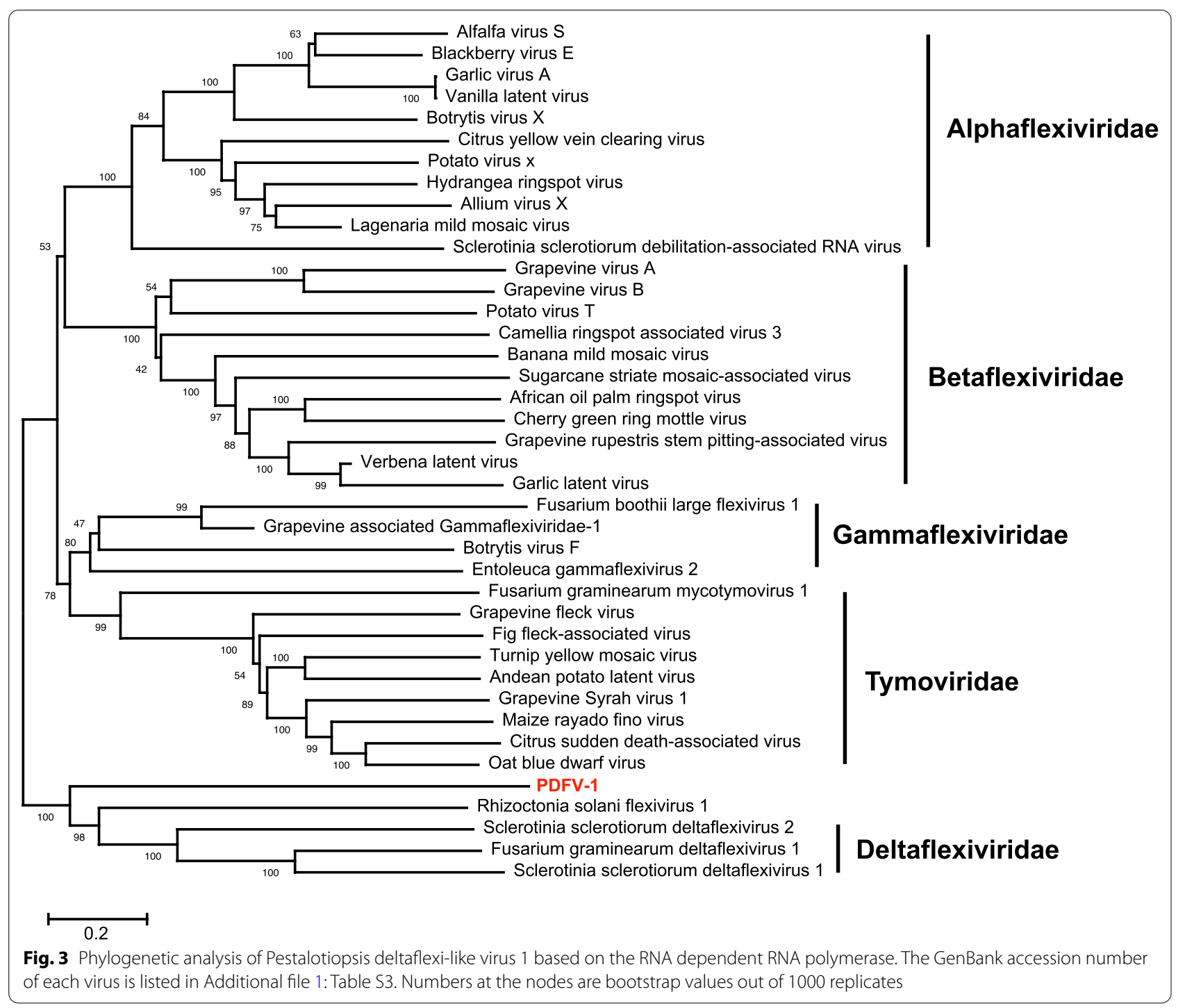

populations of both B. cinerea and S. sclerotiorum [28]. Therefore, CgOLV1 may be able to be transmitted among different fungal groups. These data suggest that the transmission of mycoviruses among different fungal species may be widely occurring in natural conditions.

Mitoviruses are a group of naked viruses with linear + ssRNA genomes, possessing one large ORF that encodes an RdRp of 2.3-3.6 kb in size [29,30], with few exceptions [31]. They are thought to replicate in mitochondria since they often use mitochondrial genetic code for the translation of RdRps [32, 33]. Two mitoviruses, PMV-1 and PMV-2, were identified in the present study, as both of them possessed the six characteristic conserved motifs of mitoviruses [19] (Additional file 1: Fig. S4). Phylogenetic analysis also supported this conclusion (Fig. 2). As both of them showed no sequence identity of $>90 \%$ to all reported mitoviruses, they should be two novel members in the genus Mitovirus.

The order Tymovirales is composed of five approved families (Alpha-, Beta-, Delta-, Gammaflexiviridae, and Tymoviridae) with viral genomes of + ssRNA, and all members contain a linear genome of 5.9-9.0 nt in length [34]. Only one possible member (PDFV-1) in this order was identified. Although the BLAST search showed that PDFV-1 was most closely related to Rhizoctonia solani flexivirus 1 , the aa sequence identity between the two viruses was low at $30 \%$. The conserved motifs in the Mtr, $\mathrm{Hel}$, and RdRp domains of PDFV-1 were similar to those of deltaflexiviruses [35, 36]. Furthermore, phylogenetic analysis showed that PDFV-1 and Rhizoctonia solani flexivirus 1 were clustered with viruses in viral family Deltaflexiviridae with $100 \%$ bootstrap support. These results suggest that PDFV-1 may be a novel member of 


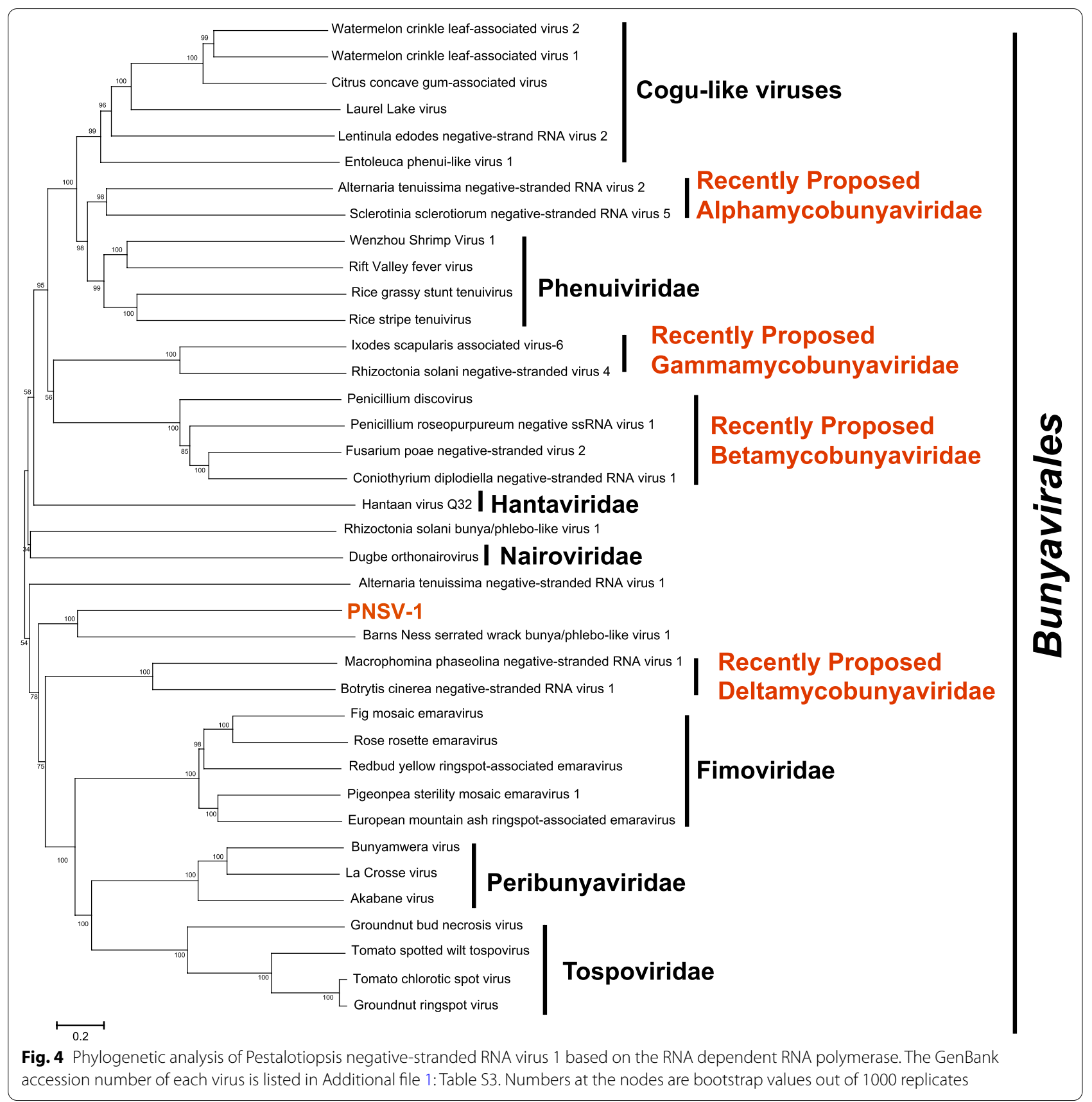

this family, but it is more distantly related to the reported deltaflexiviruses (Fig. 3).

Previous studies showed that most fungal -ss RNA viruses belonged to two orders, Mononegavirales and Bunyavirales [37]. Recent years, due to the development of metagenomics sequencing, diverse bunyaviruses have been reported in fungi, including $B$. cinerea [11], Macrophomina phaseolina [10], Lentinula edodes [38], Penicillium roseopurpureum [39], and some grapevine wood-inhabiting endophytic fungi [40]. In addition, bunyaviruses have also been detected in some oomycetes, such as Pythium polare [41] and Halophytophthora spp. [42]. A possible -ssRNA virus, PNSV-1, was identified in these Pestalotiopsis viruses. The phylogeny and conserved motif analysis indicated that PNSV-1 might belong to Bunyavirales but not Mononegavirales. PNSV-1 was firstly grouped with barns ness serrated wrack bunya/ phlebo-like virus 1 with $100 \%$ bootstrap support (Fig. 4), without showing high sequence similarity with other bunyaviruses. Therefore, PNSV-1 cannot be classified 


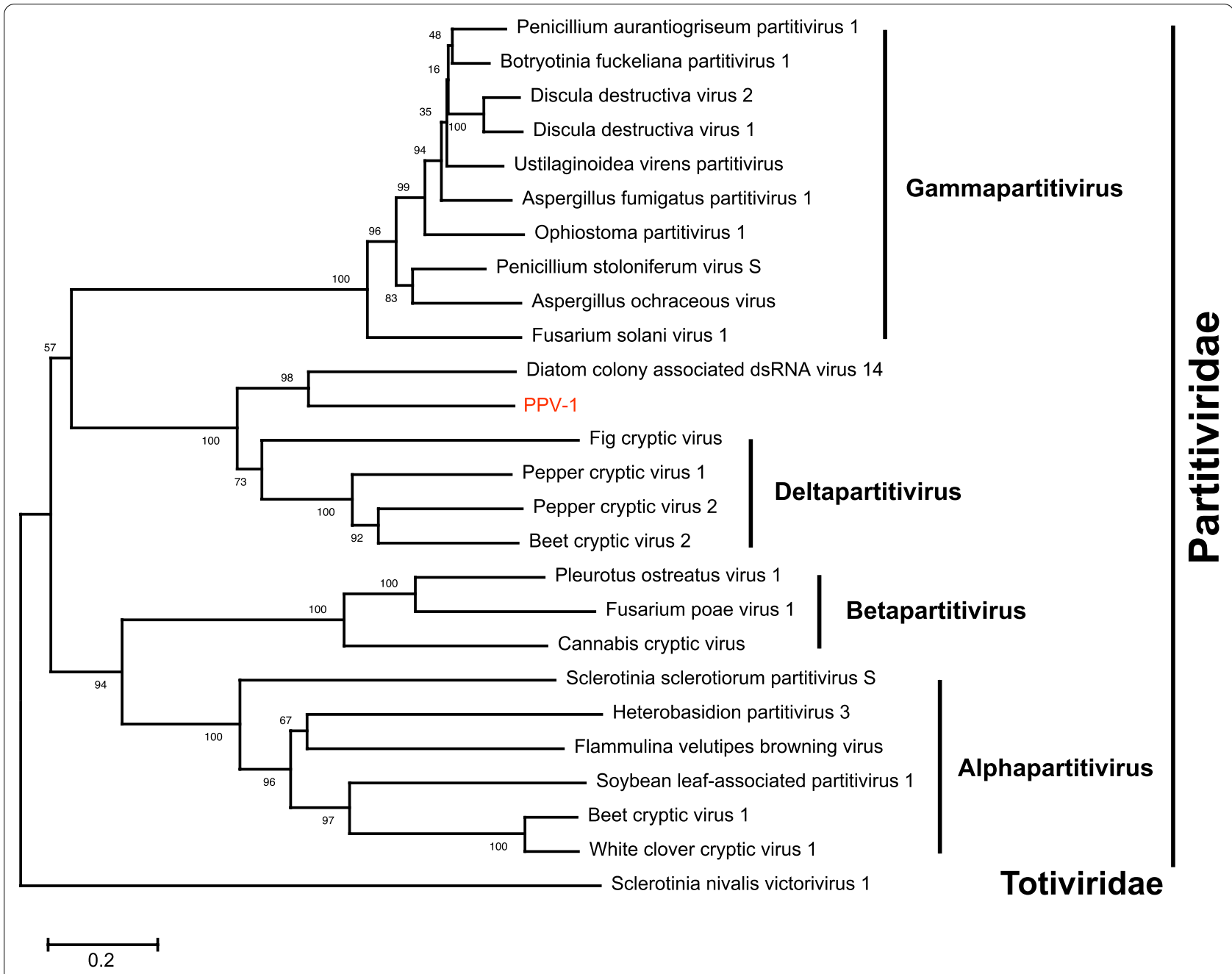

Fig. 5 Phylogenetic analysis of Pestalotiopsis partitivirus 1 based on the RNA dependent RNA polymerase. The GenBank accession number of each virus is listed in Additional file 1: Table S3. Numbers at the nodes are bootstrap values out of 1000 replicates

into any reported viral family and should belong to a new viral family within the order Bunyavirales.

Partitiviruses are a widely spread group of viruses infecting plants, fungi, and protozoa. Most partitiviruses possess two dsRNA genomes, of which one encodes the RdRp, while the other encodes the CP [43]. The family Partitiviridae contains five genera, Alphapartitivirus, Betapartitivirus, Gammapartitivirus, Deltapartitivirus, and Cryspovirus, of which alphapartitiviruses and betapartitiviruses infect both plants and fungi, whereas gammapartitiviruses and deltapartitiviruses infect only fungi and plants [43]. PPV-1 was the only identified partitivirus in the present study. Its RdRp was most closely related to diatom colony associated dsRNA virus 14 (DcV14) with a low sequence identity of $49 \%$. However, no CP sequence of PPV-1 was obtained. Phylogenetic analysis showed PPV-1 was clustered with other deltapartitiviruses with $100 \%$ bootstrap support. However, PPV-1 formed an independent branch with DcV14 in the deltapartitivirus clade, indicating that it is distantly related to the present members in this genus (Fig. 5). As the reported deltapartitiviruses only infect plants, this result indicates that deltapartitiviruses may not only infect plants but also fungi.

This is the first report showing the existence of various mycoviruses in Pestalotiopsis spp. isolated from $M$. rubra in China. In this study, we identified sequences that putatively represented the genomes of 8 viruses, many of which were nearly full-length. However, the effects of these viruses on Pestalotiopsis spp. remain unknown and needed to be investigated in further studies. Accessing virus mediated effects on Pestalotiopsis spp. will be helpful for further development of these viruses as biocontrol agents for the control of twig dieback in M. rubra. 


\section{Conclusions}

This is the first study showing the existence of various mycoviruses in Pestalotiopsis spp. isolated from $M$. rubra in China. In this study, eight mycoviruses were identified and could be assigned into five distinct lineages, including Botourmiaviridae, Narnaviridae, Partitiviridae, Tymovirales, and Bunyavirales. Within these viruses, PNSV-1 may belong to a novel viral family. Through metetranscriptomic analysis, more mycoviral groups were detected and may be tapped as biocontrol agents for fungal diseases of plants.

\section{Supplementary Information}

The online version contains supplementary material available at https://doi. org/10.1186/s12985-021-01513-3.

Additional file 1. Figure S1. RT-PCR confirmation of eight viral-like contigs in the mixed RNA sample. See Table S2 for detailed information of primers and amplicon sizes. Figure S2. Pairwise identity comparisons of botoumiaviral (a) and mitoviral (b) sequences in Pestalotiopsis strains to other known botoumiaviruses and mitoviruses. See Table S3 for detailed information of each virus. Figure S3. Conserved amino acid sequence motifs of the putative RNA-dependent RNA polymerases of Colletotrichum gloeosporioides ourmia-like virus 1 (CgOLV1), Pestalotiopsis botourmiavirus 2 (PBV-2), Pestalotiopsis botourmiavirus 3 (PBV-3) Botrytis ourmia-like virus (BOLV), Phoma matteucciicola ourmia-like virus 1 (PmOLV1), Magnaporthe oryzae ourmia-like virus (MOLV), and Rhizoctonia solani ourmia-like virus 1 (RsOLV1). The GenBank accession number of each virus is listed in Table S3. "*" indicates identical amino acid residues; and ".' indicates low chemically similar amino acid residues. Figure S4. Conserved amino acid sequence motifs of the putative RNA-dependent RNA polymerases of Pestalotiopsis mitovirus 1 (PMV-1), Pestalotiopsis mitovirus 2 (PMV-2), Cryphonectria cubensis mitovirus 2a (CcMV2a), Ophiostoma mitovirus 1a (OnuMV1a), Sclerotinia homoeocarpa mitovirus (ShMV), Ophiostoma mitovirus 3a (OnuMV1a), and Cryphonectria parasitica mitovirus 1-NB631 (CpMV1-NB631). The GenBank accession number of each virus is listed in Table S3. "*" indicates identical amino acid residues; and "'.' indicates low chemically similar amino acid residues. Figure S5. Multiple alignment of amino acid sequences of conserved domains including methyltransferase (Mtr), viral RNA Helicase (Hel), and RNA-dependent RNA polymerase (RdRp) domains of Pestalotiopsis deltaflexi-like virus 1 (PDFV-1), Sclerotinia sclerotiorum deltaflexivirus 1 (SsDFV1), Sclerotinia sclerotiorum deltaflexivirus 2 (SsDFV2), and Fusarium graminearum deltaflexivirus 1 (FgDFV1). The GenBank accession number of each virus is listed in Table S3. "** indicates identical amino acid residues; and ".' indicates low chemically similar amino acid residues. Figure S6. Conserved amino acid sequence motifs of the putative RNA-dependent RNA polymerases of Pestalotiopsis negative-stranded RNA virus 1, rose rosette emaravirus (RRV), and fig mosaic emaravirus (FMV). The GenBank accession number of each virus is listed in Table S3. "** indicates identical amino acid residues; and "'.' indicates low chemically similar amino acid residues. Figure S7. Conserved amino acid sequence motifs of the putative RNA-dependent RNA polymerases of Pestalotiopsis partitivirus 1 (PPV-1), beet cryptic virus 1 (BCV1), beet cryptic virus 2 (BCV2), beet cryptic virus 3 (BCV3), and fig cryptic virus ( $\mathrm{FCV}$ ). The GenBank accession number of each virus is listed in Table S3. "**" indicates identical amino acid residues; and "'.' indicates low chemically similar amino acid residues. Table S1. Strains used in this study. Table S2. List of PCR primers used for viral contig detection. Table S3. Viruses used for multiple sequence alignments and phylogenetic analysis.

\section{Abbreviations}

NGS: Next-generation sequencing; PDA: Potato dextrose agar; NCBI: National Center for Biotechnology Information; ORF: Open reading frame; NJ: Neighbor-joining; RdRp: RNA dependent RNA polymerase; CgOLV1: Colletotrichum gloeosporioides ourmia-like virus 1; PBV-2: Pestalotiopsis botourmiavirus 2; PMV-1: Pestalotiopsis mitovirus 1; PMV-2: Pestalotiopsis mitovirus 2; PDFV-1: Pestalotiopsis deltaflexivirus 1; PNSV-1: Pestalotiopsis negative-stranded RNA virus 1; PPV-1: Pestalotiopsis partitivirus 1; DcV14: Diatom colony associated dsRNA virus 14.

\section{Acknowledgements}

Not applicable.

\section{Authors' contributions}

Conceptualization, F.C.; methodology, F.C. and H.N.; software, Y.W.; validation, F.C., H.N. and B.Y.; investigation, F.N.; data curation, F.C. and H.N.; writingoriginal draft preparation, F.C., Z.P.; writing-review and editing, F.C. and H.N.; project administration, F.C.; funding acquisition, F.C. All authors read and approved the final manuscript.

\section{Funding}

This research is a cooperative project between Zhejiang Provincial People's Government and Chinese Academy of Forestry, Grant Number 2019SY05-01; The discipline construction of Zhejiang Academy of Agricultural Sciences, Grant Number 2018, No. 8 (2018-2023); Taizhou Science and Technology Project, Grant Number 20NY15.

\section{Availability of data and materials}

The viral sequences are available on NCBI at https://www.ncbi.nlm.nih.gov/. The raw data used for metetranscriptomic analysis are available at the Bioproject PRJNA661377 in NCBI.

\section{Ethics approval and consent to participate}

Not applicable.

\section{Consent to publication \\ Not applicable.}

\section{Competing interests}

The authors declare that they have no competing interests.

Received: 18 November 2020 Accepted: 10 February 2021

Published online: 23 February 2021

\section{References}

1. He XH, Chen LG, Chen Y, Guo CL. Review on germplasm resources of Myrica and their exploitation in China. J Fruit Sci. 2004;21:467-71.

2. Chen FY, Lu L, Ni HZ, Wang Y, Wang YG. First report of Pestalotiopsis mangiferae and P. vismiae causing twig dieback of Myrica rubra in China. Plant Dis. 2012;96:588.

3. Chen FY, Lu L, Wang DM, Wang Y, Ni HZ, Du ZG. Biological characterization and genetic diversity analysis of two species of Pestalotiopsis causing twig dieback of Myrica rubra. Eur J Plant Pathol. 2013;136:737-47.

4. Ren HY, Li G, Qi XJ, Fang L, Wang HR, Wei JG, Zhong SB. Identification and characterization of Pestalotiopsis spp. causing twig blight disease of bayberry (Myrica rubra Sieb \& Zucc.) in China. Eur J Plant Pathol. 2013;137:451-61.

5. Yang XL, Zhang JZ, Luo DQ. The taxonomy, biology and chemistry of the fungal Pestalotiopsis genus. Nat Prod Rep. 2012;29:622-41.

6. Hollings M. Viruses associated with a die-back disease of cultivated mushroom. Nature. 1962;196:962-5.

7. Rigling D, Prospero S. Cryphonectria parasitica, the causal agent of chestnut blight: invasion history, population biology and disease control. Mol Plant Pathol. 2018;19:7-20.

8. Vainio EJ, Jurvansuu J, Streng J, Rajamaki ML, Hantula J, Valkonen JPT. Diagnosis and discovery of fungal viruses using deep sequencing of small RNAs. J Gen Virol. 2015;96:714-25.

9. Marzano SY, Domier LL. Novel mycoviruses discovered from metatranscriptomics survey of soybean phyllosphere phytobiomes. Virus Res. 2016;213:332-42

10. Marzano LSY, Nelson BD, Ajayi-Oyetunde O, Bradley CA, Hughes TJ, Hartman GL, Eastburn DM, Domiera LL. Identification of diverse mycoviruses 
through metatranscriptomics characterization of the viromes of five major fungal plant pathogens. JVirol. 2016;90:6846-63.

11. Donaire L, Ayllon MA. Deep sequencing of mycovirus-derived small RNAs from Botrytis species. Mol Plant Pathol. 2017;18:1127-37.

12. Mu F, Xie JT, Cheng SF, You MP, Barbetti MJ, Jia JC, Wang QQ, Cheng JS, Fu YP, Chen T, Jiang DH. Virome Characterization of a collection of Sclerotinia sclerotiorum from Australia. Front Microbiol. 2017;8:2540.

13. Liang N, Yang D, Wu MD, Zhang J, Li GQ, Yang L. Molecular characterization of a novel botoulivirus from the phytopathogenic fungus Sclerotinia minor. Arch Virol. 2020;165:785-8.

14. Yu X, Li B, Fu YP, Jiang DH, Ghabrial SA, Li GQ, Peng YL, Xie JT, Cheng $J S$, Huang J, Yi X. A geminivirus-related DNA mycovirus that confers hypovirulence to a plant pathogenic fungus. Proc Natl Acad Sci USA. 2010;107:8387-92.

15. Li PF, Wang SC, Zhang LH, Qiu DW, Zhou XP, Guo LH. A tripartite ssDNA mycovirus from a plant pathogenic fungus is infectious as cloned DNA and purified virions. Sci Adv. 2020;6:eaay9634.

16. Muhire B, Martin DP, Brown JK, Navas-Castillo J, Moriones E, et al. A genome-wide pairwise-identity-based proposal for the classification of viruses in the genus Mastrevirus (family Geminiviridae). Arch Virol. 2013;158:1411-24.

17. Kumar S, Stecher G, Li M, Knyaz C, Tamura KMEGAX. Molecular evolutionary genetics analysis across computing platforms. Mol Biol Evol. 2018:35:1547-9.

18. Koonin EV. The phylogeny of RNA-dependent RNA polymerases of positive strand RNA viruses. J Gen Virol. 1991;72:2197-206.

19. Hong Y, Dover SL, Cole TE, Brasier CM, Buck KW. Multiple mitochondrial viruses in an isolate of the Dutch elm disease fungus Ophiostoma novoulmi. Virology. 1999;258:118-27.

20. Bruenn JAA. A Closely related group of RNA-dependent RNA polymerases from double-stranded RNA viruses. Nucleic Acids Res. 1993:21:5667-9.

21. Li CX, Zhu JZ, Gao BD, Zhu HJ, Zhou Q, Zhong J. Characterization of a novel ourmia-like mycovirus infecting Magnaporthe oryzae and implications for viral diversity and evolution. Viruses. 2019;11:223.

22. Zhou J, Wang Y, Liang X, Xie C, Liu W, Miao W, Kang Z, Zheng L. Molecular characterization of a novel ourmia-like virus infecting Phoma matteucciicola. Viruses. 2020;12:231.

23. Melzer MS, Ikeda SS. Boland GJ Interspecific transmission of doublestranded RNA and hypovirulence from Sclerotinia sclerotiorum to S. minor. Phytopathology. 2002;92:780-4.

24. Liu YC, Hillman BI, Linder-Basso D, Kaneko S, Milgroom MG. Evidence for interspecies transmission of viruses in natural populations of filamentous fungi in the genus Cryphonectria. Mol Ecol. 2003;12:1619-28.

25. Coenen A, Kevei F, Hoekstra RF. Factors affecting the spread of doublestranded RNA viruses in Aspergillus nidulans. Genet Res. 1997;69:1-10.

26. Wu MD, Jin FY, Zhang J, Yang L, Jiang DH, Li GQ. Characterization of a novel bipartite double-stranded RNA mycovirus conferring hypovirulence in the phytopathogenic fungus Botrytis porri. J Virol. 2012;86:6605-19.

27. Wu MD, Wang QJ, Zhang J, Yang L, Li GQ. A novel dsRNA mycovirus infecting the phytopathogenic fungus Botrytis squamosa. J Huazhong Agric Univ. 2014;33:39-46.

28. Hao F, Wu MD, Li G. Molecular characterization and geographic distribution of a mymonavirus in the population of Botrytis cinerea. Viruses. 2018;10:432.
29. Ohkit S, Lee Y, Nguyen Q, Ikeda K, Suzukib N, Nakayashiki H. Three ourmialike viruses and their associated RNAs in Pyricularia oryzae. Virology. 2019;534:25-35.

30. Xu ZY, Wu SS, Liu L, Cheng JS, Fu YP, Jiang DH, Xie JT. A mitovirus related to plant mitochondrial gene confers hypovirulence on the phytopathogenic fungus Sclerotinia sclerotiorum. Virus Res. 2015;197:127-36.

31. Vainio EJ. Mitoviruses in the conifer root rot pathogens Heterobasidion annosum and H. parviporum. Virus Res. 2019;271:197681.

32. Hong Y, Cole TE, Brasier CM, Buck KW. Evolutionary relationships among putative RNA-dependent RNA polymerases encoded by a mitochondrial virus-like RNA in the Dutch elm disease fungus, Ophiostoma novo-ulmi, by other viruses and virus-like RNAs and by the Arabidopsis mitochondrial genome. Virology. 1998;246:158-69.

33. Polashock JJ, Hillman Bl. A small mitochondrial double-stranded (ds) RNA element associated with a hypovirulent strain of the chestnut blight fungus and ancestrally related to yeast cytoplasmic T and W dsRNAs. Proc Natl Acad Sci USA. 1994;91:8680-4.

34. Martelli GP, Adams MJ, Kreuze JF, Dolja WV. Family Flexiviridae: a case study in virion and genome plasticity. Annu Rev Phytopathol. 2007;45:73-100.

35. Hamid MR, et al. A Novel deltaflexivirus that infects the plant fungal pathogen, Sclerotinia sclerotiorum, can be transmitted among host vegetative incompatible strains. Viruses. 2018;10:295.

36. Li KF, Zheng D, Cheng JS, Chen T, Fu YP, Jiang DH, Xie JT. Characterization of a novel Sclerotinia sclerotiorum RNA virus as the prototype of a new proposed family within the order Tymovirales. Virus Res. 2016;219:92-9.

37. Liu L, Xie JT, Cheng JS, Fu YP, Li GQ, Yi X, Jiang DH. Fungal negativestranded RNA virus that is related to bornaviruses and nyaviruses. Proc Natl Acad Sci USA. 2014;111:12205-10.

38. Lin YH, Fujita M, Chiba S, Hyodo K, Andika IB, Suzuki N, Kondo H. Two novel fungal negative-strand RNA viruses related to mymonaviruses and phenuiviruses in the shiitake mushroom (Lentinula edodes). Virology. 2019:533:125-36

39. Nerva L, Forgia M, Ciuffo M, Chitarra W, Chiapello M, Vallino M, Varesec GC, Turina M. The mycovirome of a fungal collection from the sea cucumber Holothuria polii. Virus Res. 2019:273:197737.

40. Nerva L, Turina M, Zanzotto A, Gardiman M, Gaiotti F, Gambino G, Chitarra W. Isolation, molecular characterization and virome analysis of culturable wood fungal endophytes in esca symptomatic and asymptomatic grapevine plants. Environ Microbiol. 2019;21:2886-904.

41. Sasai S, Tamura K, Tojo M, Herrero ML, Hoshino T, Ohki ST, Mochizuki T. A novel non-segmented double-stranded RNA virus from an Arctic isolate of Pythium polare. Virology. 2018;522:234-43.

42. Botella L, Janoušek J, Maia C, Jung MH, Raco M, Jung T. Marine oomycetes of the genus Halophytophthora harbor viruses related to bunyaviruses. Front Microbiol. 2020;11:1-13.

43. Nibert ML, Ghabrial NSA, Maiss E, Lesker T, Vainio EJ, Jiang DH, Suzuki N. Taxonomic reorganization of family Partitiviridae and other recent progress in partitivirus research. Virus Res. 2014;188:128-41.

\section{Publisher's Note}

Springer Nature remains neutral with regard to jurisdictional claims in published maps and institutional affiliations.

Ready to submit your research? Choose BMC and benefit from:

- fast, convenient online submission

- thorough peer review by experienced researchers in your field

- rapid publication on acceptance

- support for research data, including large and complex data types

- gold Open Access which fosters wider collaboration and increased citations

- maximum visibility for your research: over $100 \mathrm{M}$ website views per year

At BMC, research is always in progress.

Learn more biomedcentral.com/submissions 